\title{
Análise do conhecimento de alunos da educação de jovens e adultos (EJA) sobre doenças sexualmente transmissíveis
}

\author{
Xênia Larissa Motta FERNANDES ${ }^{1 *}$ \\ Maria Cláudia Mota PEDROSO ${ }^{2 *}$ \\ Luana Maria RABÊLO ${ }^{3 *}$ \\ Aline Helena da Silva CRUZ \\ Gláucia Maria CAVASIN ${ }^{5}$
}

\author{
${ }^{1}$ Graduanda em Medicina, Universidade Federal de Goiás. \\ ${ }^{2}$ Graduanda em Medicina, Universidade Federal de Goiás. \\ ${ }^{3}$ Graduanda em Medicina, Universidade Federal de Goiás. \\ ${ }^{4}$ Bióloga, Mestre em Biologia, ICB/Departamento de Morfologia/Universidade Federal de Goiás. Co-orientadora do \\ trabalho. alinehelena@hotmail.com \\ ${ }^{5}$ Bióloga, Mestre em Entomologia, Doutorado em Ciências Biológicas, ICB/Departamento de Morfologia/Universidade \\ Federal de Goiás. Orientadora do trabalho. glauciacavasin@gmail.com \\ * As autoras contribuíram igualmente no desenvolvimento do trabalho.
}

Recebido em: 30/10/2012 - Aprovado em: 20/12/2012 - Disponibilizado em: 30/12/2012

RESUMO: As políticas públicas e demais ações de promoção da saúde, relativas às DST (doenças sexualmente transmissíveis), devem atentar para quais informações ou o que as pessoas conhecem sobre DST. Dados do Boletim Epidemiológico - AIDS/DST do Ministério da saúde apresentam um total de 608.230 casos de AIDS notificados de 1980 a junho de 2011 no Brasil. E apesar das instituições de ensino serem um excelente campo de conscientização sobre as DSTs, no cotidiano das escolas há uma grande dificuldade dos professores em abordar assuntos relacionados com a sexualidade não só com adolescentes, mas também com jovens e adultos membros da EJA (Educação de jovens e adultos). O presente artigo apresenta resultados do trabalho desenvolvido com alunos da EJA pelo Projeto Sexualidade - Mitos e Verdades da UFG, que aborda temas que envolvem a sexualidade e DSTs. Foi possível verificar que a maior parte dos alunos sabe identificar pelo menos um sintoma que costuma ser relacionado às DSTs, além disso, as mulheres apresentaram maior conhecimento sobre as formas de prevenção da AIDS do que os homens, mas também foi possível verificar a falta de conhecimento dos alunos sobre informações básicas referentes a prevenção de DSTs. Estes resultados expõem a necessidade da atuação de projetos e políticas públicas relacionadas à sexualidade não só entre adolescentes, mas também entre jovens e adultos, tendo como campo aberto e carente, os estudantes da EJA.

PALAVRAS-CHAVE: DSTs. Doenças sexualmente transmissíveis. AIDS. Educação de Jovens e Adultos. EJA.

\section{Analysis of knowledge of students and the education of young adults (EJA) about diseases sexually transmitted}

\begin{abstract}
Public politics and other actions to promote health, relating to STD (sexually transmitted diseases), should pay attention to what information or what people know about STDs. Data from the Epidemiological Bulletin AIDS / STD Ministry of Health show a total of 608,230 AIDS cases were reported from 1980 to June 2011 in Brazil. And while educational institutions are excellent field awareness about STDs, in daily school there is a great difficulty for teachers in teach subjects related to sexuality not only to teens but also to young and adults members of the EYA (education of youth and adults). This article presents results of the work with the EYA students by Sexuality Project at UFG, which subjects involving sexuality and STDs. We noticed that most students can identify at least one symptom that is often related to STDs, moreover, women had greater knowledge about ways of preventing AIDS than men, but it was also possible to verify the absence of students' knowledge of basic information regarding the prevention of STDs. These results expose the need of projects and public politics related to sexuality not only among adolescents but also among youth and adults, it having the open field and needy, the students of the EYA.
\end{abstract}

KEYWORDS: STD. Sexually transmitted diseases. AIDS. EYA. Education of youth and adults. 


\section{1 - INTRODUÇÃO}

A adolescência é um período em que o ser humano vive uma das épocas em que experimenta e explora o mundo, o que pode ser motivo de conflitos e dúvidas, especialmente no que concerne ao corpo e à descoberta da sexualidade (BORUCHOVITCH, 1992). Tendo em vista as novidades e mudanças de comportamento que passam a fazer parte da vida do jovem, a vida sexual apresenta-se envolvida num risco maior, uma vez que o jovem pode se sujeitar a problemas como a gravidez não planejada e as doenças sexualmente transmissíveis (DSTs).

Mas alterações comportamentais referentes à sexualidade são observadas também em outro grupo da sociedade. Pois, o envelhecimento da população e o aumento do número de pessoas idosas, acompanhado por melhoria da qualidade de vida, vêm estimulando mudanças de comportamento relacionadas à sexualidade, proporcionando às pessoas relações afetivas mais ativas.

Pesquisa sobre comportamento da população brasileira sexualmente ativa, realizada pelo Programa Nacional de DST/AIDS, em 2003, mostrou que 67,1\% das pessoas de 50 a 59 anos e $39,2 \%$ das pessoas com 60 anos e mais de idade são sexualmente ativos, e a proporção de homens sexualmente ativos com mais de 50 anos é o dobro daquela observada entre as mulheres com a mesma idade (PAIVA et al 2004).
O aumento da atividade sexual sem proteção na população adulta confirma dados do Ministério da Saúde que vem notificando aumento no número de casos de AIDS na população com 40 anos e mais de idade. Do total de casos notificados em 1992, 15,6\% foram para homens de 40 a 49 anos, aumentando para $24,4 \%$ em 2003. Para a faixa de 50 a 59 anos, de $4,5 \%$ aumentou para $8,7 \%$ e de 60 anos e mais de $1,8 \%$ aumentou para 2,7\% no mesmo período (BOLETIM EPIDEMIOLÓGICO-AIDS, 2003).

Nos Estados Unidos, $10 \%$ dos casos de AIDS em mulheres ocorreram na faixa de 50 anos e mais, e $32 \%$ dessas mulheres tinham 60 anos e mais de idade quando foi diagnosticado, sugerindo que as infecções ocorreram na pós-menopausa (HENDERSON et al., 2004).

Diante disto, percebemos que as ações de promoção em saúde, relativas às DST, devem considerar o comportamento sexual dos indivíduos, as informações sobre suas práticas sexuais, além de atentar para quais informações ou o que as pessoas conhecem sobre DST. A compreensão sobre as práticas sexuais e o conhecimento que as pessoas têm sobre DST podem acrescentar evidências sobre os fatores de risco e de proteção em relação às doenças (CARRENO et al., 2006).

Dados apresentados no BOLETIM EPIDEMIOLÓGICO-AIDS/DST revelam que:

De 1980 a junho de 2011, no Brasil, foram notificados no 
Sinan, declarados no SIM e registrados no Siscel/Siclom um total de 608.230 casos de AIDS, sendo $343.095(56,4 \%)$ na Região Sudeste; $123.069 \quad(20,2 \%)$ na Região Sul; 78.686 (12,9\%) na Região Nordeste; 35.116 (5,8\%) na Região Centro-Oeste; e 28.248 $(4,7 \%)$ na Região Norte.

Tendo em vista os problemas relacionados às doenças sexualmente transmissíveis (DSTs), o Projeto Sexualidade - Mitos e Verdades, uma iniciativa de professores da Universidade Federal de Goiás que visa romper barreiras entre a Universidade e as escolas, tem levado a estudantes de ensino fundamental e médio noções relacionadas à descoberta da sexualidade, os riscos e envolvidos, a consciência sobre as mudanças fisiológicas vivenciadas e esclarecimentos sobre métodos contraceptivos e preventivos das DSTs, adequadas a realidade de cada grupo escolar trabalhado.

Dentre estes grupos escolares está também o desafio em desenvolver o projeto com estudantes da Educação de jovens e adultos (EJA), caracterizados geralmente por não terem concluído os estudos na adolescência e entrado na fase adulta sem muitos dos conhecimentos, relacionados saúde, que são aprendidos rotineiramente nas escolas. Mas, além disso,

é preciso considerar que a
motivação e a mobilização para os
estudos na idade adulta
relacionam-se não só às
exigências de formação da vida
cotidiana e à quantidade e
qualidade das oportunidades de
atendê-las, mas também aos

\begin{abstract}
horizontes de mudança socioeconômica e nas estruturas de poder, o que depende de políticas participativas e redistributivas que transcendem a esfera educacional. (PIERRO, 2010)
\end{abstract}

Além disso, nota-se no cotidiano das escolas uma grande dificuldade dos professores em abordar assuntos relacionados com a sexualidade não só quando o trabalho é desenvolvido com adolescentes, considerados inexperientes na vida sexual, mas também com os adultos, considerados experientes. Por isso, o Projeto Sexualidade também realiza pesquisas e palestras sobre o conhecimento geral de temas relacionados à sexualidade que visam auxiliar os professores no desenvolvimento deste tema que também faz parte do conteúdo escolar.

O presente trabalho apresenta parte dos resultados deste projeto, os quais foram obtidos através do trabalho desenvolvido com alunos da EJA, que responderam um questionário diferenciado que aborda assuntos específicos relacionados à DSTs, visando uma nova abordagem dos temas que envolvem a sexualidade, a conscientização deste grupo de estudantes da existência destas doenças, bem como a necessidade de prevenção e tratamento das mesmas. Sendo necessário considerar o fato de que

Assume-se, como desafio, a
constituição de práticas
educativas que contemplem as
necessidades próprias desse
público jovem e adulto. Para além
do acesso à escolarização, é
fundamental refletir sobre sua
forma e conteúdo, considerando




\begin{tabular}{|c|c|c|}
\hline espe & idade & dessa \\
\hline nodalidade & de & ensino. \\
\hline $\begin{array}{l}\text { (MOREIRA } \\
\text { 2011) }\end{array}$ & $\&$ & FERREIRA \\
\hline
\end{tabular}

\section{METODOLOGIA}

Público alvo: O presente trabalho foi desenvolvido com 62 alunos estudantes da rede estadual de ensino, da região central de Goiânia-GO que cursam do sexto ao nono ano da EJA no período noturno.

Projeto Sexualidade - Mitos e verdades: Através do projeto sexualidade, graduandos do curso de Medicina, orientados por professores da UFG, ministram palestras a respeito de DSTs. Utilizando recursos didáticos variados como vídeos, pôster, moldes e textos, os alunos enfatizam as formas de transmissão, necessidade de prevenção e tratamento das DSTs. As palestras têm duração média de 25 minutos. Sendo que a primeira etapa de contato com os alunos ocorre através da aplicação de um questionário diagnóstico sobre DSTs.

Questionário: Visando a adequação das palestras a realidade de cada grupo escolar os membros do projeto formularam um questionário direcionado para jovens e adultos estudantes da EJA. O questionário contém questões diversificadas sobre DSTs, que são respondidas pelos alunos antes das palestras de forma individual, anônima e espontânea. O questionário possui questões amplas que focam desde o significado da sigla DST, exemplos de DSTs, até sintomas e formas de transmissão das mesmas. A partir das informações obtidas nos questionários, os membros do projeto organizam as palestras que são ministradas em uma nova visita a comunidade escolar.

\section{RESULTADOS E DISCUSSÃO}

Os resultados aqui apresentados são embasados nas informações fornecidas pelos 62 estudantes da EJA da rede Estadual de ensino do Estado de Goiás. Vale ressaltar que as entrevistas foram realizadas em um momento do planejamento da disciplina de Ciências em que o (a) professor (a) ainda não havia ministrado, naquele período letivo, conteúdos relacionados ao tema proposto neste artigo.

No questionário respondido pelos estudantes, de forma individual, as duas primeiras questões se preocuparam em obter dados que identificassem os indivíduos analisados por idade e sexo. Sendo que, dos 62 alunos questionados 29 são do sexo masculino $(46,77 \%)$ e 33 do sexo feminino $(53,23 \%)$ (Figura - 1), todos em idade fértil, variando de 15 a 48 anos. De forma que a diferença na idade do indivíduo mais jovem para o mais velho é de 33 anos. 


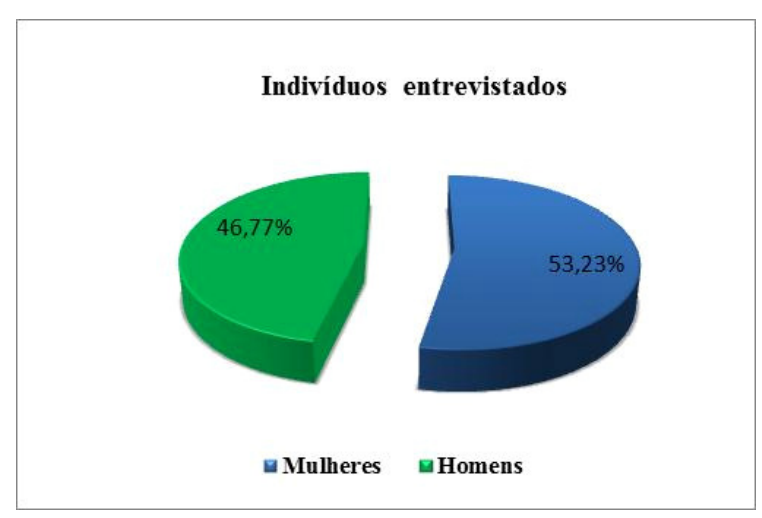

Figura - 1: Indivíduos entrevistados. $\mathrm{O}$ total de indivíduos entrevistados foi considerado como $100 \%$, depois foi calculada a porcentagem equivalente de homens e mulheres.

Além disso, 66,13\% dos entrevistados são considerados adultos (Figura - 2), considerando que a estrutura etária de uma população costuma ser dividida em três faixas: os jovens, que são do nascimento até 19 anos; os adultos, dos 20 anos até 59 anos e os idosos, que vai dos 60 anos em diante (VESENTINI, 1998).

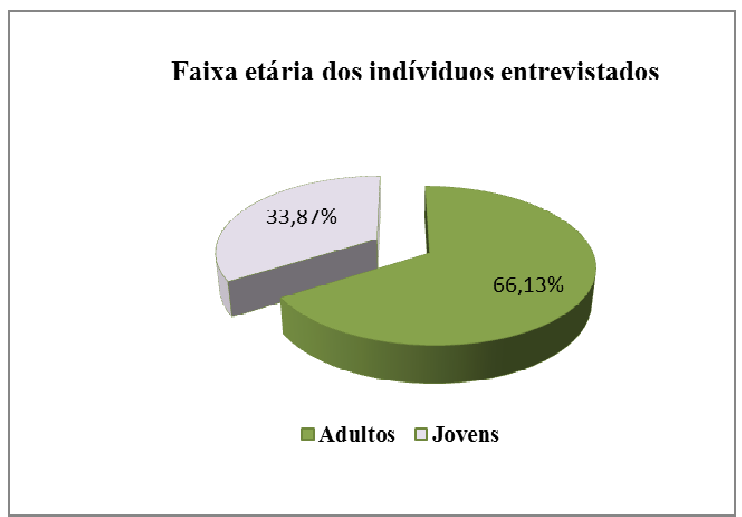

Figura - 2: Faixa etária dos indivíduos entrevistados. O total de indivíduos entrevistados foi considerado como $100 \%$ e faixa etária destes, classificada em adultos e jovens.

Esses resultados nos mostram o quão heterogêneo é o grupo avaliado, e a necessidade de políticas públicas específicas para este público (EJA), que é formado por jovens que há pouco entraram na puberdade e adultos que já são avós quiçá bisavós. E como bem dito por PIERRO (2010):

Para que as políticas públicas
possam conferir materialidade a
concepções mais apropriadas de
alfabetização e educação básica
de qualidade (o que implica
também articular devidamente
oportunidades de qualificação
profissional e acesso às
tecnologias da comunicação e
informação), é preciso ampliar o
financiamento destinado à EJA e
reverter a situação de despreparo
e desvalorização profissional dos
educadores que a ela se
dedicam.(...) Ao lado do
estabelecimento de condições
mínimas de trabalho profissional,
a formação de educadores é um
dos grandes desafios a serem
encarados pelas políticas
educacionais nos próximos anos.

A partir da terceira pergunta os entrevistavam responderam questões relacionadas às doenças sexualmente transmissíveis (DSTs), utilizando apenas o conhecimento pessoal, obtido não só em informações divulgadas através de políticas públicas do governo, mas também pela mídia e o aprendizado escolar, obtido principalmente nas aulas de Ciências, em outros períodos letivos.

Baseando no fato de que a maior parte dos cartazes e propagandas relacionadas à prevenção de doenças que são transmitidas através do ato sexual utiliza o termo DST, perguntamos se os alunos sabiam o significado deste ou se pelo menos já ouviram falar do mesmo. Através das respostas verificamos que em um total de $100 \%$, $31,03 \%$ dos homens e $24,24 \%$ das mulheres 
desconheciam o termo DST (Figura - 3, porção vermelha) e $31,03 \%$ dos homens e $24,24 \%$ das mulheres tinham conhecimento do termo (Figura - 3, porção azul). Dos que já ouviram falar no termo DST, $15 \%$ dos homens e 15,38\% das mulheres não souberam definir o significado das siglas.

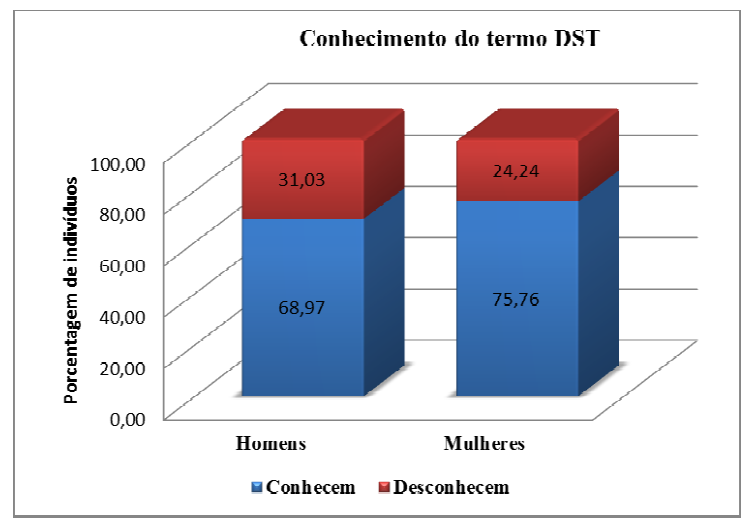

Figura - 3: Porcentagem de indivíduos entrevistados que conhecem o do termo DST. O total de homens foi considerado $100 \%$ e o total de mulheres considerado $100 \%$.

Após a explicação do termo para todos os alunos, o questionário foi concluído pelos entrevistados tendo como resultado o fato de aproximadamente $24 \%$ destes não saberem nenhum exemplo de DST. Sendo que, 75,86\% dos homens e $75,75 \%$ das mulheres sabem pelo menos um exemplo, vale ressaltar que verificamos que muitos alunos não sabiam citar mais do que um exemplo de DST.

A outra preocupação foi em relação à saúde dos indivíduos e se eles sabiam identificar sintomas que podem estar relacionados a estas doenças. Então, uma questão de múltipla escolha foi formulada com oito sintomas diferentes: dor de cabeça, corrimento, verrugas na região genital, ardor ao urinar, tosse, coceira na região genital, falta de ar, dor nas costas. Devendo o aluno assinalar todos os itens que indicavam sintomas de DST.

A grande maioria dos entrevistados $93,93 \%$ das mulheres e $86,20 \%$ dos homens soube identificar pelo menos um sintoma que costuma ser relacionado à DST (Figura - 4).

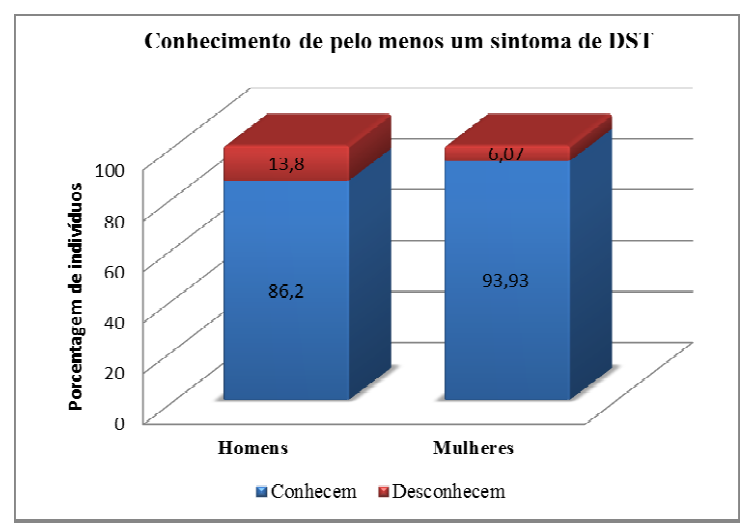

Figura - 4: Porcentagem de indivíduos entrevistados que conhecem pelo menos um sintoma de DST. O total de homens foi considerado $100 \%$ e o total de mulheres considerado $100 \%$.

O que deixa claro que apesar de muitos não conhecerem ou entenderem a diversidade de doenças relacionadas ao ato sexual eles sabem identificar modificações em seus corpos que indicam que algo está errado, logo, que podem ter alguma doença.

Dentre as DSTs de conhecimento dos alunos, a mais citada foi a AIDS/SIDA (Síndrome da Imuno Deficiência Adquirida), sendo que, $100 \%$ das mulheres e $96,55 \%$ dos homens sabem pelo menos uma forma de contágio do vírus da AIDS. Mas infelizmente o conhecimento sobre as formas de prevenção desta doença, que acaba servindo para a grande maioria das DSTs, não foi tão elevado. 
Somente $65,51 \%$ dos homens e $81,81 \%$ das mulheres tinham conhecimento de pelo menos uma forma de prevenção da doença (Figura $5)$.

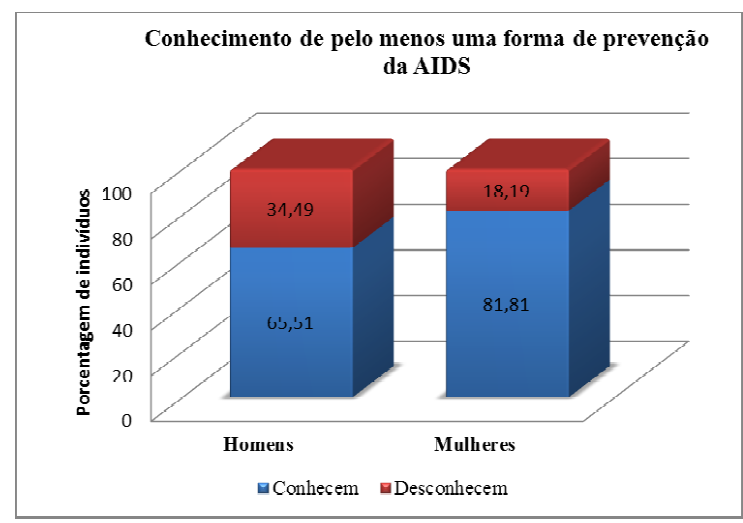

Figura - 5: Porcentagem de indivíduos entrevistados que conhecem pelo menos uma forma de prevenção da AIDS. O total de homens foi considerado $100 \%$ e o total de mulheres considerado $100 \%$.

Essa diferença no maior conhecimento de sintomas, contágio e prevenção de DSTs, por parte das mulheres do que dos homens, provavelmente está relacionada com a cultura destas em serem mais presentes nos consultórios médicos, bem como de atentarem mais às mudanças que ocorrem no próprio corpo. Além disso, o interesse das mulheres em utilizarem o método contraceptivo mais adequado pode ser um diferencial que lhes proporciona um contato contínuo com médicos ginecologistas que lhes proporcionam esclarecimentos sobre as DSTs.

A falta de conhecimento de métodos preventivos de AIDS/DSTs por parte dos estudantes é altamente preocupante. Dados disponíveis no site do Ministério da Saúde mostram que:

\begin{abstract}
As doenças sexualmente transmissíveis (DST) são consideradas como um dos problemas de saúde pública mais comuns em todo o mundo. Em ambos os sexos, tornam o organismo mais vulnerável a outras doenças, inclusive a AIDS, além de terem relação com a mortalidade materna e infantil. No Brasil, as estimativas da Organização Mundial de Saúde (OMS) de infecções de transmissão sexual na população sexualmente ativa, a cada ano, são: Sífilis: 937.000, Gonorreia: 1.541.800, Clamídia: 1.967.200, Herpes genital: 640.900 e HPV: 685.400. (DST no Brasil)
\end{abstract}

Além disso, o BOLETIM EPIDEMIOLÓGICO - AIDS/DST (2012) apresentado pelo ministério da saúde, que "De 1980 a junho de 2011, no Brasil, foram notificados 608.230 casos de aids. Em 2010 foram notificados 34.218 novos casos, com taxa de incidência nacional de 17,9/100.000 habitantes e razão de sexo de 1,7 novos casos em homens para cada caso em mulheres."

\section{CONCLUSÕES}

A banalização do sexo e da sexualidade na mídia e no cotidiano dificulta a tarefa de educar, de associar sexo a afeto, responsabilidade e promoção da saúde e bem estar. Em síntese, a tarefa de educar e transmitir valores nos dias atuais tem sido um desafio para famílias e principalmente para os educadores. (OLIVI et al., 2008).

Embora os dados sejam obtidos no presente trabalho sejam de apenas uma parcela dos estudantes que se encontram 
matriculados em toda rede estadual de Goiás, o conhecimento apresentado pela parcela da população estudada pode refletir características comuns àqueles que frequentam a EJA das escolas públicas do Estado, ou mesmo do País, devido ao perfil de estudantes que ingressam nesta modalidade de ensino.

Os resultados deixam clara a necessidade da atuação de projetos e políticas públicas relacionadas à sexualidade não só entre adolescentes, mas também entre jovens e adultos. E a melhor forma de atuação destes projetos continua sendo no ambiente escolar, pois, além da grande dificuldade cultural em se falar de sexo e sexualidade em casa, muitos alunos, que também são pais, não possuem conhecimento adequado sobre as DSTs e consequentemente não conseguem orientar adequadamente seus filhos a respeito desse tema. Além disso, o fato da EJA ser formada por uma grande parcela de adultos torna-a um campo disseminador de informações.

Apesar das políticas públicas relacionadas à DSTs se voltarem intensamente para os adolescentes, faz-se necessário mais políticas públicas que alcancem os pais destes adolescentes. Além de pensarmos e cuidarmos dos adultos do futuro é necessário também os cuidados com os adultos do presente e idosos do futuro.

\section{AGRADECIMENTOS}

Agradecemos a Universidade Federal de Goiás, toda comunidade escolar que participou deste trabalho e todos os demais membros do Projeto Sexualidade - Mitos e Verdades, sem os quais seria impossível a obtenção dos dados aqui apresentados.

\section{REFERÊNCIA BIBLIOGRÁFICA}

BOLETIM EPIDEMIOLÓGICO-AIDS ( $1^{\mathrm{a}}$ a $52^{\mathrm{a}}$ semanas epidemiológicas). Ministério da Saúde; 2003.

\section{BOLETIM EPIDEMIOLÓGICO -} AIDS/DST. (Ano VIII - no $1-27^{\mathrm{a}}$ a $52^{\mathrm{a}}$ semanas epidemiológicas - julho a dezembro de 2010; Ano VIII - n $1-01^{\mathrm{a}}$ a $26^{\mathrm{a}}$ - semanas epidemiológicas - janeiro a junho de 2011).

Ministério da Saúde. Ano VIII n ${ }^{\circ} 01.2012$

BORUCHOVITCH, E. Fatores associados a não utilização de anticoncepcionais na adolescência. Rev Saúde Pública; v. 26: p.437-43. 1992

CARRENO, I.; COSTA, J.S.D.; Uso de preservativos nas relações sexuais: estudo de base populacional. Rev Saúde Pública; v.40(4): p.720-26; agosto 2006

DST no Brasil. Ministério da saúde. Diponível em: http://www.aids.gov.br/pagina/dst-no-brasil. Acesso em: 30 de outubro de 2012.

HENDERSON, S.J.; BERNSTEIN, L.B.; GEORGE, D.M.; DOYLE, J.P.; PARANJAPE, A.S.; CORBIE-SMITH, G. Older women and HIV: how much do they know and where are they getting their information? J Am Geriatr Soc. September; v. 51(9): p. 1549-53. 2004

MOREIRA, A. F.; FERREIRA, L. A. G.2 Abordagem temáticac e contextos de vida em uma prática educativa em ciências e biologia 
na EJA. Ciência \& Educação, v. 17, n. 3, p. 603-624, 2011.

OLIVI, M. SANTANA, R. G.MATHIAS, T. A. F. Comportamento, conheciemnto e percepção de risco sobre doenças sexualemnte transmissíveis em um grupo de pessoas com 50 anos e mais de idade. Rev Latino-am Enfermagem. V.16(4); julho-agosto. 2008

PAIVA V, VENTURI G, FRANÇA JRI, LOPES F. Uso de preservativos: Pesquisa Nacional MS/IBOPE 2003. [relatório online]. [Acesso 4 Ago 2004]. Disponível em: URL:

http://www.aids.gov.br/final/biblioteca/ibope_ 2003/briefing2.doc.

PIERRO, M. C. A Educação de Jovens e adultos no plano nacional de educação: avaliação, desafios e perspectivas. Educ. Soc., Campinas, v. 31, n. 112, p. 939-959, jul.-set. 2010

VESENTINI, Jose William. Brasil: sociedade \& espaço: geografia do Brasil. 28. ed. reform. e atual. São Paulo: Atica, 1998. 352p. ISBN 8508070233 